\title{
Minima of quasisuperminimizers
}

\author{
Anders Björn \\ Department of Mathematics, Linköping University, \\ SE-58183 Linköping, Sweden; anders.bjorn@liu.se \\ Jana Björn \\ Department of Mathematics, Linköping University, \\ SE-58183 Linköping, Sweden; jana.bjorn@liu.se \\ Riikka Korte \\ Department of Mathematics, P.O. Box 68 (Gustaf Hällströmin katu 2b), \\ FI-00014 University of Helsinki, Finland; riikka.korte@helsinki.fi
}

\begin{abstract}
Let $u_{i}$ be a $Q_{i}$-quasisuperminimizer, $i=1,2$, and $u=\min \left\{u_{1}, u_{2}\right\}$, where $1 \leq Q_{1} \leq Q_{2}$. Then $u$ is a quasisuperminimizer, and we improve upon the known upper bound (due to Kinnunen and Martio) for the optimal quasisuperminimizing constant $Q$ of $u$. We give the first examples with $Q>Q_{2}$, and show that in general $Q>Q_{2}$ whenever $Q_{1}>1$. We also study the blowup of the quasisuperminimizing constant in pasting lemmas.
\end{abstract}

Key words and phrases: Blowup, metric space, nonlinear potential theory, pasting lemma, quasiminimizer, quasisuperharmonic function, quasisuperminimizer.

Mathematics Subject Classification (2010): Primary: 31C45; Secondary: 31E05, 35J60.

\section{Introduction}

Let $1<p<\infty$ and let $\Omega \subset \mathbf{R}^{n}$ be a nonempty open set. A function $u \in W_{\text {loc }}^{1, p}(\Omega)$ is a $Q$-quasiminimizer, $Q \geq 1$, in $\Omega$ if

$$
\int_{\varphi \neq 0}|\nabla u|^{p} d x \leq Q \int_{\varphi \neq 0}|\nabla(u+\varphi)|^{p} d x
$$

for all $\varphi \in W_{0}^{1, p}(\Omega)$. A function $u$ is a $Q$-quasisuper(sub)minimizer if (1.1) holds for all nonnegative (nonpositive) $\varphi \in W_{0}^{1, p}(\Omega)$.

Quasiminimizers were introduced by Giaquinta and Giusti [15], [16] as a tool for a unified treatment of variational integrals, elliptic equations and quasiregular mappings on $\mathbf{R}^{n}$. They realized that De Giorgi's method could be extended to quasiminimizers, obtaining, in particular, local Hölder continuity. DiBenedetto and Trudinger [14] proved the Harnack inequality for quasiminimizers, as well as weak Harnack inequalities for quasisub- and quasisuperminimizers. A little later, Ziemer [35] gave a Wiener-type criterion sufficient for boundary regularity for quasiminimizers, and Tolksdorf [33] obtained a Caccioppoli inequality and a convexity result for quasiminimizers. The results in [14]-[16] and [35] were extended to metric spaces by Kinnunen-Shanmugalingam [22] and J. Björn [10] in the beginning of this century, see also A. Björn-Marola [8]. Soon afterwards, Kinnunen-Martio [21] 
showed that quasiminimizers have an interesting potential theory, in particular they introduced quasisuperharmonic functions, which are related to quasisuperminimizers in a similar way as superharmonic functions are related to supersolutions. The theory of quasi(super)minimizers has been further studied in [1]-[5], [7], [9], [11][13], [17], [19], [20], [23]-[32] and [34].

It is well known that the minimum of two superharmonic functions is again superharmonic. This property is used extensively e.g. in balayage and in the Perron method for solving the Dirichlet problem. For quasisuperminimizers, KinnunenMartio [21] showed the following similar result. (We formulate it in $\mathbf{R}^{n}$, but it is valid also in metric measure spaces, see Section 2. The same holds for Theorems 1.2 and 1.4.)

Theorem 1.1. (Kinnunen-Martio [21]) Let $u_{j}$ be a $Q_{j}$-quasisuperminimizer, $j=$ 1,2. Then $\min \left\{u_{1}, u_{2}\right\}$ is a $\min \left\{Q_{1} Q_{2}, Q_{1}+Q_{2}\right\}$-quasisuperminimizer.

The blowup of the quasisuperminimizing constant in this result is the main focus of this paper. Our first result is the following better upper bound.

Theorem 1.2. Let $u_{i}$ be a $Q_{i}$-quasisuperminimizer in $\Omega$ for $i=1,2$. Then $u=$ $\min \left\{u_{1}, u_{2}\right\}$ is a $\bar{Q}$-quasisuperminimizer in $\Omega$, where

$$
\bar{Q}= \begin{cases}1, & \text { if } Q_{1}=Q_{2}=1, \\ \left(Q_{1}+Q_{2}-2\right) \frac{Q_{1} Q_{2}}{Q_{1} Q_{2}-1}, & \text { otherwise }\end{cases}
$$

In particular, if $Q_{1}=Q_{2}$, then $\bar{Q}=2 Q_{1}^{2} /\left(Q_{1}+1\right)$.

Note that when $Q_{1}, Q_{2}>1$, we always have the following bounds for $\bar{Q}$ in (1.2):

$$
Q_{1}+Q_{2}-2<\bar{Q}<Q_{1}+Q_{2}-1<\min \left\{Q_{1} Q_{2}, Q_{1}+Q_{2}\right\}
$$

This means that we obtain a better blowup constant than Kinnunen-Martio [21] whenever $Q_{1}, Q_{2}>1$.

In the converse direction it is clear that $u$ cannot (in general) have a better quasisuperminimizing constant than $\max \left\{Q_{1}, Q_{2}\right\}$ (and thus already Theorem 1.1 is optimal if $Q_{1}=1$ or $Q_{2}=1$ ). As far as we know, there have so far not been any examples showing that some blowup is indeed possible. We construct such examples in Section 3. In particular, we prove the following result.

Theorem 1.3. Let $p>1$ and $1<Q_{1} \leq Q_{2}$. Then there exist functions $u_{1}$ and $u_{2}$ on $(0,1) \subset \mathbf{R}$ such that $u_{j}$ is a $Q_{j}$-quasisuperminimizer in $(0,1), j=1,2$, but $\min \left\{u_{1}, u_{2}\right\}$ is not a $Q_{2}$-quasisuperminimizer in $(0,1)$.

We also obtain estimates for the blowup in the quasisuperminimizing constant. In Section 4 we give an upper bound for the blowup when taking a minimum of three quasisuperminimizers, which is better than iterating Theorem 1.2.

Another result with a blowup in the quasisuperminimizing constant is the following pasting lemma.

Theorem 1.4. (Björn-Martio [9, Theorem 4.1]) Assume that $\Omega_{1} \subset \Omega_{2} \subset \mathbf{R}^{n}$ are open and that $u_{j}$ is a $Q_{j}$-quasisuperminimizer in $\Omega_{j}, j=1,2$. Let

$$
u= \begin{cases}u_{2}, & \text { in } \Omega_{2} \backslash \Omega_{1}, \\ \min \left\{u_{1}, u_{2}\right\}, & \text { in } \Omega_{1} .\end{cases}
$$

If $u \in W_{\mathrm{loc}}^{1, p}\left(\Omega_{2}\right)$, then $u$ is a $Q_{1} Q_{2}$-quasisuperminimizer in $\Omega_{2}$. 
In Theorems 5.1 and 5.2 we show that the blowup constant $Q_{1} Q_{2}$ is optimal in this result. There is also a similar pasting lemma for quasisuperharmonic functions in Björn-Martio [9, Theorem 5.1] and our optimality result applies also to this case, see Remark 5.3.

Yet another result with a blowup of the quasisuperminimizing constant is the reflection principle by Martio [26, Theorem 3.1]. In one dimension (i.e. on $\mathbf{R}$ ) he obtained a better result in Theorem 4.1 in [26]. The blowup constant in the latter result was subsequently improved upon by Uppman [34, Lemma 2.8], who also showed that his constant is the best possible.

Acknowledgement. The first two authors were supported by the Swedish Research Council. The third author was supported by the Academy of Finland, grant no. 250403. Part of this research was done while the third author visited Linköping University in 2009, and while all three authors visited Institut Mittag-Leffler in the autumn of 2013. They want to thank the institute for the hospitality.

\section{An upper bound for the blowup}

In this section we are going to prove Theorem 1.2. Let us however first discuss some consequences and generalizations of it.

Definition 2.1. A function $u: \Omega \rightarrow(-\infty, \infty]$ is $Q$-quasisuperharmonic in $\Omega$ if $u$ is not identically $\infty$ in any component of $\Omega, \min \{u, k\}$ is a $Q$-quasisuperminimizer in $\Omega$ for every $k \in \mathbf{R}$, and $u$ is lower semicontinuously regularized, i.e.

$$
u(x)=\operatorname{ess} \liminf _{y \rightarrow x} u(y) \quad \text { for } x \in \Omega
$$

This definition is equivalent to Definition 7.1 in Kinnunen-Martio [21], see Theorem 7.10 in [21]. Using this definition we obtain the following corollary of Theorem 1.2 .

Corollary 2.2. Let $u_{i}$ be a $Q_{i}$-quasisuperharmonic function in $\Omega$ for $i=1,2$. Then $u=\min \left\{u_{1}, u_{2}\right\}$ is $\bar{Q}$-quasisuperharmonic in $\Omega$, where $\bar{Q}$ is given by (1.2).

We have formulated Theorem 1.2 and Corollary 2.2 on (unweighted) $\mathbf{R}^{n}$, but they have direct counterparts valid in complete metric spaces equipped with doubling measures supporting a $p$-Poincaré inequality (and thus also on weighted $\mathbf{R}^{n}$ with a $p$-admissible weight), see Björn-Björn [6] for more on the metric space theory (note that Appendix $\mathrm{C}$ therein gives a short survey on quasiminimizers).

Below we have chosen to give an $\mathbf{R}^{n}$ proof of Theorem 1.2. However, it carries over verbatim to metric spaces, with the trivial modifications that $|\nabla u|$ is replaced by the minimal $p$-weak upper gradient $g_{u}$ (and similarly for the other gradients) and $d x$ is replaced by $d \mu$. Note that $g_{u}=|\nabla u|$ on unweighted and weighted $\mathbf{R}^{n}$, see Appendices A.1 and A.2 in [6].

Proof of Theorem 1.2. Let $0 \leq \varphi \in W_{0}^{1, p}(\Omega)$ be arbitrary and set

$$
\begin{aligned}
A & =\{x \in \Omega: \varphi(x)>0\}, \\
A_{1} & =\left\{x \in A: u_{1}(x)<u_{2}(x)\right\}, \\
A_{2} & =\left\{x \in A: u_{2}(x)<u_{1}(x)\right\}, \\
A_{0} & =\left\{x \in A: v(x)>\max \left\{u_{1}(x), u_{2}(x)\right\}\right\},
\end{aligned}
$$

where $v=u+\varphi$. Note that $A=A_{1} \cup A_{2} \cup A_{0}$, though not pairwise disjointly. 
We may assume that $\int_{A}|\nabla u|^{p} d x<\infty$, as otherwise (1.1) holds trivially, since the triangle inequality together with the fact that $\varphi \in W^{1, p}(\Omega)$ implies that

$$
\left(\int_{A}|\nabla(u+\varphi)|^{p} d x\right)^{1 / p} \geq\left(\int_{A}|\nabla u|^{p} d x\right)^{1 / p}-\left(\int_{A}|\nabla \varphi|^{p} d x\right)^{1 / p}=\infty .
$$

Let $\varphi_{1}=\left(\min \left\{u_{2}, v\right\}-u_{1}\right)_{+}$and note that $0 \leq \varphi_{1} \leq \varphi$, which implies that $\varphi_{1} \in$ $W_{0}^{1, p}(\Omega)$. The $Q_{1}$-quasisuperminimizing property of $u_{1}$ yields

$$
\int_{\varphi_{1}>0}\left|\nabla u_{1}\right|^{p} d x \leq Q_{1} \int_{\varphi_{1}>0}\left|\nabla\left(u_{1}+\varphi_{1}\right)\right|^{p} d x
$$

Note that $\varphi_{1}(x)>0$ if and only if $u_{2}(x)>u_{1}(x)$ and $u_{1}(x)+\varphi(x)=v(x)>u_{1}(x)$, which in turn holds exactly when $x \in A_{1}$. Moreover,

$$
u_{1}+\varphi_{1}= \begin{cases}u_{2}, & \text { in } A_{1} \cap A_{0}, \\ v, & \text { in } A_{1} \backslash A_{0} .\end{cases}
$$

Multiplying (2.1) by $\left(Q_{2}-1\right)$ then gives

$$
\left(Q_{2}-1\right) \int_{A_{1}}\left|\nabla u_{1}\right|^{p} d x \leq Q_{1}\left(Q_{2}-1\right)\left(\int_{A_{1} \cap A_{0}}\left|\nabla u_{2}\right|^{p} d x+\int_{A_{1} \backslash A_{0}}|\nabla v|^{p} d x\right) .
$$

Similarly, using $\varphi_{2}=\left(\min \left\{u_{1}, v\right\}-u_{2}\right)_{+} \in W_{0}^{1, p}(\Omega)$ and the $Q_{2}$-quasisuperminimizing property of $u_{2}$ we obtain (after multiplication with $\left(Q_{1}-1\right)$ ),

$$
\left(Q_{1}-1\right) \int_{A_{2}}\left|\nabla u_{2}\right|^{p} d x \leq Q_{2}\left(Q_{1}-1\right)\left(\int_{A_{2} \cap A_{0}}\left|\nabla u_{1}\right|^{p} d x+\int_{A_{2} \backslash A_{0}}|\nabla v|^{p} d x\right) .
$$

Next, let $\widetilde{\varphi}_{j}=\left(v-u_{j}\right)_{+}, j=1,2$. Since $0 \leq \widetilde{\varphi}_{j} \leq \varphi$, we have $\widetilde{\varphi}_{j} \in W_{0}^{1, p}(\Omega)$ and (1.1) with $u_{j}$ and $\widetilde{\varphi}_{j}$ gives

$$
\int_{\widetilde{\varphi}_{j}>0}\left|\nabla u_{j}\right|^{p} d x \leq Q_{j} \int_{\widetilde{\varphi}_{j}>0}\left|\nabla\left(u_{j}+\widetilde{\varphi}_{j}\right)\right|^{p} d x
$$

Now, $\widetilde{\varphi}_{1}(x)>0$ if and only if $\min \left\{u_{1}(x), u_{2}(x)\right\}+\varphi(x)>u_{1}(x)$, which is equivalent to $x \in A$ (i.e. $\varphi(x)>0$ ) and $u_{2}(x)+\varphi(x)>u_{1}(x)$. This in turn holds exactly if $x \in A_{1}$ or $u_{2}(x) \leq u_{1}(x)<u_{2}(x)+\varphi(x)=v(x)$, i.e. when $x \in A_{1} \cup A_{0}$. Similarly, $\widetilde{\varphi}_{2}(x)>0$ if and only if $x \in A_{2} \cup A_{0}$.

Since $u_{j}+\widetilde{\varphi}_{j}=v$ whenever $\widetilde{\varphi}_{j}>0$, the inequalities in (2.4) give

$$
Q_{2}\left(Q_{1}-1\right) \int_{A_{1} \cup A_{0}}\left|\nabla u_{1}\right|^{p} d x \leq Q_{1} Q_{2}\left(Q_{1}-1\right) \int_{A_{1} \cup A_{0}}|\nabla v|^{p} d x
$$

and

$$
Q_{1}\left(Q_{2}-1\right) \int_{A_{2} \cup A_{0}}\left|\nabla u_{2}\right|^{p} d x \leq Q_{1} Q_{2}\left(Q_{2}-1\right) \int_{A_{2} \cup A_{0}}|\nabla v|^{p} d x,
$$

where we have also multiplied by $Q_{2}\left(Q_{1}-1\right)$ and $Q_{1}\left(Q_{2}-1\right)$, respectively.

Next, we shall sum up the inequalities (2.2), (2.3), (2.5) and (2.6) as follows. The first term in the right-hand side of (2.2) can be subtracted from the left-hand side of (2.6), leaving

$$
\begin{aligned}
Q_{1}\left(Q_{2}-1\right) \int_{\left(A_{2} \cup A_{0}\right) \backslash A_{1}}\left|\nabla u_{2}\right|^{p} d x \\
=Q_{1}\left(Q_{2}-1\right)\left(\int_{A_{2}}\left|\nabla u_{2}\right|^{p} d x+\int_{A_{0} \backslash\left(A_{1} \cup A_{2}\right)}\left|\nabla u_{2}\right|^{p} d x\right)
\end{aligned}
$$


therein. Since $u=u_{2}$ in $A \backslash A_{1} \supset A_{2}$, adding this to the left-hand side of $(2.3)$ results in

$$
\begin{aligned}
\left(Q_{1}\left(Q_{2}-1\right)\right. & \left.+\left(Q_{1}-1\right)\right) \int_{A_{2}}|\nabla u|^{p} d x+Q_{1}\left(Q_{2}-1\right) \int_{A_{0} \backslash\left(A_{1} \cup A_{2}\right)}|\nabla u|^{p} d x \\
& =\left(Q_{1} Q_{2}-1\right) \int_{A_{2}}|\nabla u|^{p} d x+Q_{1}\left(Q_{2}-1\right) \int_{A_{0} \backslash\left(A_{1} \cup A_{2}\right)}|\nabla u|^{p} d x
\end{aligned}
$$

as $\left|\nabla u_{2}\right|$ 's contribution to the left-hand side of the final sum.

Similarly, subtracting the first term in the right-hand side of (2.3) from the left-hand side of (2.5), and adding the left-hand side of (2.2) contributes with

$$
\left(Q_{1} Q_{2}-1\right) \int_{A_{1}}|\nabla u|^{p} d x+Q_{2}\left(Q_{1}-1\right) \int_{A_{0} \backslash\left(A_{1} \cup A_{2}\right)}|\nabla u|^{p} d x
$$

to the left-hand side of the final sum. Since $Q_{1}\left(Q_{2}-1\right)+Q_{2}\left(Q_{1}-1\right) \geq Q_{1} Q_{2}-1$, summing up (2.7) and (2.8) shows that the left-hand side in the final sum will be

$$
\begin{gathered}
\left(Q_{1} Q_{2}-1\right) \int_{A_{1} \cup A_{2}}|\nabla u|^{p} d x+\left(Q_{1}\left(Q_{2}-1\right)+Q_{2}\left(Q_{1}-1\right)\right) \int_{A_{0} \backslash\left(A_{1} \cup A_{2}\right)}|\nabla u|^{p} d x \\
\geq\left(Q_{1} Q_{2}-1\right) \int_{A_{1} \cup A_{2} \cup A_{0}}|\nabla u|^{p} d x .
\end{gathered}
$$

We now turn to the right-hand side of the sum of (2.2), (2.3), (2.5) and (2.6). The remaining term in the right-hand side of $(2.2)$ is

$$
Q_{1}\left(Q_{2}-1\right) \int_{A_{1} \backslash A_{0}}|\nabla v|^{p} d x \leq Q_{1} Q_{2}\left(Q_{2}-1\right) \int_{A_{1} \backslash A_{0}}|\nabla v|^{p} d x
$$

which together with the right-hand side of (2.6) contributes with

$$
Q_{1} Q_{2}\left(Q_{2}-1\right) \int_{A_{1} \cup A_{2} \cup A_{0}}|\nabla v|^{p} d x
$$

to the right-hand side of the final sum. Similarly, the remaining term in the righthand side of (2.3) together with the right-hand side of (2.5) gives

$$
Q_{1} Q_{2}\left(Q_{1}-1\right) \int_{A_{1} \cup A_{2} \cup A_{0}}|\nabla v|^{p} d x
$$

in the right-hand side of the final sum.

As $A_{1} \cup A_{2} \cup A_{0}=A=\{x \in \Omega: \varphi(x)>0\}$, we have thus obtained

$$
\left(Q_{1} Q_{2}-1\right) \int_{\varphi>0}|\nabla u|^{p} d x \leq Q_{1} Q_{2}\left(Q_{1}+Q_{2}-2\right) \int_{\varphi>0}|\nabla v|^{p} d x
$$

Division by $Q_{1} Q_{2}-1$ concludes the proof of Theorem 1.2. (If $Q_{1}=Q_{2}=1$, the result follows from Theorem 1.1.)

\section{Lower bounds for the blowup}

Consider two quasisuperminimizers defined on some open set $\Omega$. More precisely let $u_{j}$ be a $Q_{j}$-quasisuperminimizer in $\Omega, j=1,2$. Also let $u=\min \left\{u_{1}, u_{2}\right\}$ and assume that $Q_{1} \leq Q_{2}$. Theorem 1.1 then shows that $u$ is also a quasisuperminimizer, and it gives an upper bound on the optimal quasisuperminimizer constant $Q$ for $u$ (in terms of $Q_{1}$ and $Q_{2}$ only). In Theorem 1.2 we improved upon this upper bound. 
As far as we know, there have not been any examples showing that the optimal $Q$ can be greater than $Q_{2}$. It is obvious that one cannot do any better than $Q_{2}$ in general (just consider the cases when $u_{1} \geq u_{2}$ in $\Omega$ ). Note also that if $Q_{1}=1$, then Theorem 1.1 shows that $u$ is a $Q_{2}$-quasisuperminimizer, and hence the constants in Theorems 1.1 and 1.2 are sharp in this case.

In this section we will give several examples of pairs of quasisuperminimizers such that their minimum has a blowup of the quasisuperminimizer constant, i.e. in the notation above we get $Q>Q_{2}$. Even though the best (largest) bounds come just from one such example we feel that it can be of interest to mention several different examples as they may add a little to the knowledge on quasisuperminimizers.

Let us already now mention that in all our examples, the functions $u_{1}$ and $u_{2}$ will not only be quasisuperminimizers, but will in fact be quasiminimizers (with the same optimal constants) as well as subminimizers (i.e. 1-quasisubminimizers).

We will also prove Theorem 1.3, i.e. that whenever $Q_{1}>1$, then there are examples showing that one can have $Q>Q_{2}$ and thus that $\max \left\{Q_{1}, Q_{2}\right\}$ is an upper bound only when $Q_{1}=1$.

Our examples will all be on $\mathbf{R}$. The reason for this is that this is almost the only case when one can actually calculate optimal quasiminimizers and their constants. As far as we know, the only higher-dimensional quasi(super)minimizers for which their optimal quasi(super)minimizer constant has been determined, and is strictly larger than 1, are the power-type quasi(super)minimizers studied in Björn-Björn [5].

The easiest example of a blowup in the quasisuperminimizing constant is perhaps the following. (It was incidentally also the first example we discovered.)

Example 3.1. Let $p=2$,

$$
u_{1}(x)=\left\{\begin{array}{ll}
\frac{2}{3} x, & 0 \leq x \leq \frac{1}{2}, \\
\frac{4}{3} x-\frac{1}{3}, & \frac{1}{2} \leq x \leq 1,
\end{array} \quad \text { and } \quad u_{2}(x)= \begin{cases}\frac{5}{6} x, & 0 \leq x \leq \frac{4}{5} \\
\frac{5}{3} x-\frac{2}{3}, & \frac{4}{5} \leq x \leq 1\end{cases}\right.
$$

Then $u_{1}$ and $u_{2}$ are $\frac{9}{8}$-quasisuperminimizers (with $\frac{9}{8}=1.125$ being the optimal constant), by Theorem 5.4 below. We will call functions such as $u_{1}$ and $u_{2}$ onecorner functions.

Let $u=\min \left\{u_{1}, u_{2}\right\}$. Note that $u_{1}(x)=u_{2}(x)$ for $x=0, \frac{2}{3}, 1$. Then

$$
\int_{0}^{1}\left(u^{\prime}\right)^{2} d x=\frac{1}{2}\left(\frac{2}{3}\right)^{2}+\left(\frac{2}{3}-\frac{1}{2}\right)\left(\frac{4}{3}\right)^{2}+\left(\frac{4}{5}-\frac{2}{3}\right)\left(\frac{5}{6}\right)^{2}+\frac{1}{5}\left(\frac{5}{3}\right)^{2}=\frac{7}{6} .
$$

Comparison with $v(x)=x$ shows that $u$ is not a $Q$-quasisuperminimizer for any $Q<\frac{7}{6}=1.1666 \ldots$. The upper bounds given by Theorems 1.1 and 1.2 are

$$
\frac{81}{64}=1.265625 \text { and } \quad \frac{81}{68}=1.191176 \ldots
$$

With $Q_{1}=Q_{2}=\frac{9}{8}$ and $p=2$ this example has been optimized, i.e. $u_{1}$ and $u_{2}$ are one-corner functions with quotient between the slopes $\gamma=2$ and the choices of their corner points have been optimized to get as large blowup as possible. For $p=2$ it is a rather straightforward (although a bit lengthy) calculation to do this optimization by hand even for a general $Q=Q_{1}=Q_{2}$, and it leads to the lower bound $\frac{4}{3} Q-\frac{1}{3}$. We omit the details as we find better lower bounds below.

For other values of $p$ such optimization becomes more laborious, and we decided to do some such calculations using Maple 16. Some obtained values, correctly rounded to the nearest digit, are shown in Table 1 . These calculations suggest that for a given $Q=Q_{1}=Q_{2}$ the lower bounds increase with $p$, but the dependence on $p$ is very small (much smaller than we had expected). 


\begin{tabular}{|l|l|l|c|c|}
\hline \multicolumn{1}{|c|}{$Q$} & \multicolumn{1}{|c|}{$p=1.2$} & \multicolumn{1}{c|}{$p=2$} & $p=100$ & $\begin{array}{c}\text { Upper bound } \\
2 Q^{2} /(Q+1)\end{array}$ \\
\hline 1.001 & 1.001333193 & 1.001333333 & 1.001333353 & 1.001500250 \\
\hline 1.01 & 1.013319341 & 1.013333333 & 1.013335243 & 1.015024876 \\
\hline 1.125 & 1.164635987 & 1.166666667 & 1.166948556 & 1.191176471 \\
\hline 2 & 2.254420532 & 2.333333333 & 2.346323188 & 2.666666667 \\
\hline 10 & 11.80468177 & 13 & 13.34762304 & 18.18181818 \\
\hline 100 & 118.9796468 & 133 & 139.1598599 & 198.0198020 \\
\hline
\end{tabular}

Table 1.

Remark 3.2. Even though Example 3.1 has been optimized it should be possible, by considering more general piecewise linear functions and optimizing their parameters, to obtain better results, possibly even reaching the optimal constant for fixed $p, Q_{1}$ and $Q_{2}$, at least when the result is specialized to $\mathbf{R}$. Here, Theorem 4.1 in Martio [27] (which can also be found as Theorem C.2 in [6]) might be of help since it makes it possible to approximate quasiminimizers by other (e.g. piecewise linear) functions with almost the same quasiminimizing constant.

Another necessary ingredient would be a good control of the best quasiminimizing constant of such functions. Lemmas 2 and 8 in Martio-Sbordone [31] show that the quasiminimizing constant is at most $\left(\sup \left|u^{\prime}\right| / \inf \left|u^{\prime}\right|\right)^{p-1}$. In particular, all strictly increasing continuous piecewise linear functions (with finitely many corners) are quasiminimizers, but the best constant is not easy to obtain. Our Proposition 5.14 below is a partial step in that direction.

The above considerations open up for further numerical investigations of the blow up. We will not pursue this route as the following approach gives good lower bounds.

Definition 3.3. If $u$ is a $Q$-quasiminimizer in $\Omega \subset \mathbf{R}$ we say that $u$ has the maximal p-energy allowed by $Q$ on an interval $I \subset \Omega$ if

$$
\int_{I}\left|u^{\prime}\right|^{p} d x=Q \int_{I}\left|v^{\prime}\right|^{p} d x=Q \frac{(u(b)-u(a))^{p}}{(b-a)^{p-1}}
$$

where $v$ is the minimizer in $I$ with boundary values $v=u$ on $\partial I$, i.e.

$$
v(x)=u(a)+\frac{u(b)-u(a)}{b-a}(x-a),
$$

where $a<b$ are the end points of $I$.

Example 3.4. For $\alpha>1-1 / p$ and $x \in[0,1]$ let $v_{\alpha}(x)=x^{\alpha}$. Theorem 6.2 in Björn-Björn [5] with $n=1$ and $p>1$ implies that $v_{\alpha}$ is a $Q_{\alpha}$-quasiminimizer in $(0,1)$, where

$$
Q_{\alpha}=\frac{\alpha^{p}}{p(\alpha-1)+1}
$$

is optimal. In fact, if $1-1 / p<\alpha \leq 1$, then $v_{\alpha}$ is a superminimizer and a $Q_{\alpha^{-}}$ quasisubminimizer, while for $\alpha \geq 1, v_{\alpha}$ is a subminimizer and a $Q_{\alpha}$-quasisuperminimizer in $(0,1)$.

A simple calculation also shows that for every $x_{0} \in(0,1)$,

$$
\int_{0}^{x_{0}}\left(v_{\alpha}^{\prime}\right)^{p} d x=\int_{0}^{x_{0}} \alpha^{p} x^{p(\alpha-1)} d x=\frac{\alpha^{p} x_{0}^{p(\alpha-1)+1}}{p(\alpha-1)+1}=Q_{\alpha} \int_{0}^{x_{0}}\left(x_{0}^{\alpha-1}\right)^{p} d x
$$


where the latter integral is the $p$-energy of the linear segment from the origin to the point $\left(x_{0}, v_{\alpha}\left(x_{0}\right)\right)$. Thus, for every $x_{0} \in(0,1], v_{\alpha}$ has the maximal $p$-energy in $\left(0, x_{0}\right)$ allowed by $Q_{\alpha}$.

Note that, given $Q>1$, there are exactly two exponents $1-1 / p<\alpha^{\prime}<1<\alpha$ such that $Q=Q_{\alpha}=Q_{\alpha^{\prime}}$. This is easily shown by differentiating (3.1) and noting that the derivative is negative for $\alpha<1$ and positive for $\alpha>1$, and that $Q_{\alpha} \rightarrow \infty$ as $\alpha \rightarrow 1-1 / p$ and as $\alpha \rightarrow \infty$. We let

$$
u_{Q}(x)=x^{\alpha} \quad \text { and } \quad \bar{u}_{Q}(x)=1-(1-x)^{\alpha^{\prime}} .
$$

Then $u_{Q}(0)=\bar{u}_{Q}(0)=0$ and $u_{Q}(1)=\bar{u}_{Q}(1)=1$. Note that both $u_{Q}$ and $\bar{u}_{Q}$ are subminimizers and $Q$-quasisuperminimizers in $(0,1)$. Moreover, $u_{Q}$ has the maximal $p$-energy allowed by $Q$ on each interval $\left(0, x_{0}\right)$, while $\bar{u}_{Q}$ has the maximal $p$-energy allowed by $Q$ on each interval $\left(x_{0}, 1\right)$.

We can now use the functions $u_{Q}$ and $\bar{u}_{Q}$ above to prove Theorem 1.3.

Proof of Theorem 1.3. By Theorem 1.2, the function $u:=\min \left\{u_{Q_{1}}, \bar{u}_{Q_{2}}\right\}$ is a quasisuperminimizer in $(0,1)$ with a quasisuperminimizing constant given by (1.2). We shall show that $u$ is not a $Q_{2}$-quasisuperminimizer. To do this, it suffices to show that the $p$-energy

$$
\int_{0}^{1}\left(u^{\prime}\right)^{p} d x>Q_{2}
$$

Since $\bar{u}_{Q_{2}}$ is a subminimizer in $(0,1)$ (by Theorem 6.2 in [5]), we have that

$$
\int_{0}^{x_{0}}\left(u_{Q_{1}}^{\prime}\right)^{p} d x>\int_{0}^{x_{0}}\left(\bar{u}_{Q_{2}}^{\prime}\right)^{p} d x
$$

where the strict inequality follows from the uniqueness of solutions to obstacle problems (see e.g. Theorem 7.2 in [6]) and from the fact that $u_{Q_{1}}<\bar{u}_{Q_{2}}$ in a set of positive measure. Hence

$$
\int_{0}^{1}\left(u^{\prime}\right)^{p} d x>\int_{0}^{1}\left(\bar{u}_{Q_{2}}^{\prime}\right)^{p} d x=Q_{2}
$$

which finishes the proof.

Theorem 1.3 shows that in general there is a blow up in the quasisuperminimizing constant when taking minimum of two quasisuperminimizers, but it does not give any quantitative estimate of the blow up. Next, we shall give some lower bounds for the blow up.

Given $Q_{1}, Q_{2}>1$, let $1-1 / p<\alpha_{2}<1<\alpha_{1}$ be such that $Q_{1}=Q_{\alpha_{1}}, Q_{2}=Q_{\alpha_{2}}$ and $u_{Q_{1}}$ and $\bar{u}_{Q_{2}}$ are the corresponding quasiminimizers. Let $x_{0}$ be the unique number in $(0,1)$ such that $u_{Q_{1}}\left(x_{0}\right)=\bar{u}_{Q_{2}}\left(x_{0}\right)$, i.e. the unique solution of the equation

$$
x_{0}^{\alpha_{1}}+\left(1-x_{0}\right)^{\alpha_{2}}=1 \text {. }
$$

(To see that there is a unique solution, consider $w=\bar{u}_{Q_{2}}-u_{Q_{1}}$ and note that $w(0)=w(1)=0$. Since $w^{\prime}(0)>0$ and $w^{\prime}(1)=\infty$, there is at least one $x \in(0,1)$ such that $w(x)=0$. Next, a simple calculation shows that $w^{\prime}(x)=0$ if and only if

$$
v(x):=x^{\beta}(1-x)=\left(\frac{\alpha_{1}}{\alpha_{2}}\right)^{1 /\left(\alpha_{2}-1\right)}, \quad \text { where } \beta=\frac{\alpha_{1}-1}{1-\alpha_{2}}>0 .
$$

As $v(0)=v(1)=0$ and $v(x)$ attains its maximum at (and only at) $x=\beta /(\beta+1)$ we see that there are at most two solutions to $w^{\prime}(x)=0$, and thus there can be at most one solution to (3.4), which must lie in between those two local extrema of $w$. 


\begin{tabular}{|l|c|c|c|c|c|}
\hline \multicolumn{1}{|c|}{$Q$} & $p=1.2$ & $p=2$ & $p=100$ & $\begin{array}{c}\text { Upper bound } \\
2 Q^{2} /(Q+1)\end{array}$ & $\begin{array}{c}\widetilde{Q} \text { in }(3.9) \\
\text { for } p=2\end{array}$ \\
\hline 1.001 & 1.001480628 & 1.001480660 & 1.001480665 & 1.001500250 & 1.001373803 \\
\hline 1.01 & 1.014821935 & 1.014825154 & 1.014825593 & 1.015024876 & 1.013873175 \\
\hline 1.125 & 1.187625011 & 1.188100103 & 1.188165836 & 1.191176471 & 1.180555556 \\
\hline 2 & 2.599606519 & 2.619135721 & 2.622161265 & 2.666666667 & 2.601317394 \\
\hline 10 & 17.45294063 & 17.67321156 & 17.72170691 & 18.18181818 & 17.66438145 \\
\hline 100 & 195.7168148 & 196.3948537 & 196.5955633 & 198.0198020 & 196.3936712 \\
\hline
\end{tabular}

Table 2 .

The $p$-energy of $u=\min \left\{u_{Q_{1}}, \bar{u}_{Q_{2}}\right\}$ is then (by comparing with the $p$-energies of the linear segments connecting the origin, the point $\left(x_{0}, x_{0}^{\alpha_{1}}\right)$ and $\left.(1,1)\right)$

$$
\int_{0}^{1}\left(u^{\prime}\right)^{p} d x=Q_{1} x_{0}^{p\left(\alpha_{1}-1\right)+1}+Q_{2}\left(1-x_{0}\right)^{p\left(\alpha_{2}-1\right)+1}=: \widetilde{Q} .
$$

Here we have used that both $u_{Q_{1}}$ and $\bar{u}_{Q_{2}}$ have the maximal energies allowed by $Q_{1}$ and $Q_{2}$ in the respective intervals.

Note that $x_{0}$ is uniquely determined by $Q_{1}$ and $Q_{2}$ (through $\alpha_{1}$ and $\alpha_{2}$ ) and thus $\widetilde{Q}$ depends only on $Q_{1}$ and $Q_{2}$ (and on $p$ ). Comparing this $p$-energy with the $p$-energy of the linear function $u_{1}(x)=x$ shows that $\widetilde{Q}$ is a lower bound for the quasisuperminimizing constant of $u$. We would therefore like to estimate $\widetilde{Q}$.

The lower bounds in Table 2 have been obtained by letting Maple 16 evaluate $\widetilde{Q}$ for some values of $Q:=Q_{1}=Q_{2}$ and are compared with the upper bound obtained in Theorem 1.2. Note that these lower bounds are considerably larger, and much closer to the upper bounds, than those in Table 1.

Our next aim is to obtain more explicit estimates of $\widetilde{Q}$. Calculating $\widetilde{Q}$ in (3.5) involves first solving the equation (3.1) twice for $\alpha$, so that $Q_{1}=Q_{\alpha_{1}}$ and $Q_{2}=Q_{\alpha_{2}}$ as above, then finding $0<x_{0}<1$ such that $x_{0}^{\alpha_{1}}+\left(1-x_{0}\right)^{\alpha_{2}}=1$, and finally evaluating $\widetilde{Q}$ for the obtained values of $\alpha_{1}, \alpha_{2}$ and $x_{0}$. This can be done numerically but not analytically (not even for $p=2$ ).

A somewhat weaker, but more explicit, estimate for $\widetilde{Q}$ can be obtained in the following way. Let $x_{1} \in(0,1)$ be such that $u_{Q_{1}}\left(x_{1}\right)=\alpha_{2} x_{1}$, i.e. $x_{1}=\alpha_{2}^{1 /\left(\alpha_{1}-1\right)}$. Since $u_{Q_{1}}(0)=0, \bar{u}_{Q_{2}}^{\prime}(0)=\alpha_{2}$ and both $u_{Q_{1}}$ and $\bar{u}_{Q_{2}}$ are convex, we have that

$$
\bar{u}_{Q_{2}}(x)>\alpha_{2} x>u_{Q_{1}}(x) \text { for all } x \in\left(0, x_{1}\right) .
$$

In particular, $x_{1}<x_{0}$.

As $\bar{u}_{Q_{2}}$ is a subminimizer in $(0,1)$ and $\bar{u}_{Q_{2}}>\max \left\{u_{Q_{1}}, \alpha_{2} x\right\}$ in $\left(0, x_{0}\right)$, we then obtain (using also that $u_{Q_{1}}\left(x_{0}\right)=\bar{u}_{Q_{2}}\left(x_{0}\right)$ )

$$
\int_{0}^{x_{0}}\left(\bar{u}_{Q_{2}}^{\prime}\right)^{p} d x<\int_{0}^{x_{1}} \alpha_{2}^{p} d x+\int_{x_{1}}^{x_{0}}\left(u_{Q_{1}}^{\prime}\right)^{p} d x
$$

where the strict inequality follows as in (3.3) from the uniqueness of solutions to obstacle problems. From the fact that $u_{Q_{1}}$ has the maximal $p$-energy allowed by $Q_{1}$ on the interval $\left(0, x_{1}\right)$ we can conclude that

$$
\int_{0}^{x_{0}}\left(u_{Q_{1}}^{\prime}\right)^{p} d x=\int_{0}^{x_{1}}\left(u_{Q_{1}}^{\prime}\right)^{p} d x+\int_{x_{1}}^{x_{0}}\left(u_{Q_{1}}^{\prime}\right)^{p} d x=Q_{1} \int_{0}^{x_{1}} \alpha_{2}^{p} d x+\int_{x_{1}}^{x_{0}}\left(u_{Q_{1}}^{\prime}\right)^{p} d x .
$$


Together with (3.6) this yields

$$
\begin{aligned}
\widetilde{Q}-Q_{2} & =\int_{0}^{1}\left(\left(u^{\prime}\right)^{p}-\left(\bar{u}_{Q_{2}}^{\prime}\right)^{p}\right) d x=\int_{0}^{x_{0}}\left(u_{Q_{1}}^{\prime}\right)^{p} d x-\int_{0}^{x_{0}}\left(\bar{u}_{Q_{2}}^{\prime}\right)^{p} d x \\
& >\left(Q_{1}-1\right) \int_{0}^{x_{1}} \alpha_{2}^{p} d x=\left(Q_{1}-1\right) \alpha_{2}^{p} x_{1}=\left(Q_{1}-1\right) \alpha_{2}^{p+1 /\left(\alpha_{1}-1\right)} .
\end{aligned}
$$

(This gives another proof of Theorem 1.3.) A similar argument shows that

$$
\widetilde{Q}-Q_{1}>\left(Q_{2}-1\right) \alpha_{1}^{p}\left(1-x_{2}\right)=\left(Q_{2}-1\right) \alpha_{1}^{p+1 /\left(\alpha_{2}-1\right)},
$$

where $x_{2} \in(0,1)$ is the solution of $\bar{u}_{Q_{2}}\left(x_{2}\right)=1-\alpha_{1}\left(1-x_{2}\right)$, i.e. $1-x_{2}=\alpha_{1}^{1 /\left(\alpha_{2}-1\right)}$. Note that $x_{2}>x_{0}$. Depending on the particular values of $p, Q_{1}$ and $Q_{2}$, one of (3.7) and (3.8) may be better than the other.

For $p=2$, when $\alpha_{1}$ and $\alpha_{2}$ can be explicitly calculated in terms of $Q_{1}$ and $Q_{2}$, we get after simplification (and for $Q_{1} \leq Q_{2}$ ) that the blow up is at least the maximum of

$$
\begin{aligned}
& \widetilde{Q}-Q_{2}>\left(Q_{1}-1\right)\left(Q_{2}+\sqrt{Q_{2}^{2}-Q_{2}}\right)^{1-\sqrt{Q_{1} /\left(Q_{1}-1\right)}} \\
& \widetilde{Q}-Q_{2}>\left(Q_{1}-1\right)\left(Q_{2}-\sqrt{Q_{2}^{2}-Q_{2}}\right)^{1+\sqrt{Q_{1} /\left(Q_{1}-1\right)}}
\end{aligned}
$$

For the values considered in Tables 1 and 2, the first estimate above is quite close to those in Table 2 and better than those in Table 1.

For $p \neq 2$, we cannot obtain such explicit expressions. However, using Remark 5.10 and (5.6) below we can write

$$
\alpha_{1}=\frac{p-1}{p} \frac{\gamma_{1}^{p}-1}{\gamma_{1}^{p-1}-1} \quad \text { and } \quad \alpha_{2}=\frac{p-1}{p} \frac{\gamma_{2}^{p}-1}{\gamma_{2}^{p}-\gamma_{2}}
$$

in terms of the quotients $\gamma_{1}$ and $\gamma_{2}$ associated with $Q_{1}$ and $Q_{2}$ as in (5.4) by means of Proposition 5.5 below. A direct calculation then gives

$$
\begin{aligned}
& \alpha_{1}^{p+1 /\left(\alpha_{2}-1\right)}=\left(\frac{p\left(\gamma_{1}^{p-1}-1\right)}{(p-1)\left(\gamma_{1}^{p}-1\right)}\right)^{\frac{p(p-1)\left(\gamma_{2}-1\right)}{\gamma_{2}^{p}-1-p\left(\gamma_{2}-1\right)}}, \\
& \alpha_{2}^{p+1 /\left(\alpha_{1}-1\right)}=\left(\frac{(p-1)\left(\gamma_{2}^{p}-1\right)}{p\left(\gamma_{2}^{p}-\gamma_{2}\right)}\right)^{\frac{p(p-1) \gamma_{1}^{p-1}\left(\gamma_{1}-1\right)}{p \gamma_{1}^{p-1}\left(\gamma_{1}-1\right)-\left(\gamma_{1}^{p}-1\right)}} .
\end{aligned}
$$

In particular, for $p=2$ and $Q_{1}=Q_{2}=Q$ (and thus $\gamma_{1}=\gamma_{2}=\gamma$ ), these formulas simplify to

$$
\alpha_{1}^{p+1 /\left(\alpha_{2}-1\right)}=\left(\frac{2}{\gamma+1}\right)^{2 /(\gamma-1)},
$$

which is increasing with respect to $\gamma$ and has limit $1 / e$ as $\gamma \rightarrow 1+$, while

$$
\alpha_{2}^{p+1 /\left(\alpha_{1}-1\right)}=\left(\frac{\gamma+1}{2 \gamma}\right)^{2 \gamma /(\gamma-1)}=\left(1-\frac{\gamma-1}{2 \gamma}\right)^{2 \gamma /(\gamma-1)}<\frac{1}{e}
$$

for all $\gamma>1$. Thus $\alpha_{2}^{p+1 /\left(\alpha_{1}-1\right)}<1 / e<\alpha_{1}^{p+1 /\left(\alpha_{2}-1\right)}<1$ for all $\gamma>1$, and hence

$$
\widetilde{Q}>Q+(Q-1) / e
$$

in this case, which is better than the estimate $\frac{4}{3} Q-\frac{1}{3}=Q+\frac{1}{3}(Q-1)$ in Example 3.1, but worse than (3.9). 


\section{An upper bound for three (or more) functions}

It is possible to get estimates for the quasisuperminimizing constant for the minimum of several quasisuperminimizers by iteratively using the estimate for the minimum of two functions. The obtained estimate often depends on the order in which the minima are taken. This suggests that better estimates could be obtained, if we directly consider the minimum of all of the involved functions and as in the proof of Theorem 1.2 use all the information that is available from the fact that all the functions are quasisuperminimizers with the original constants.

To estimate the quasisuperminimizer constant for the minimum $u$ of $N$ quasisuperminimizers $u_{i}$, let $0 \leq \varphi \in W_{0}^{1, p}(\Omega)$ be arbitrary and set $v=u+\varphi$. For each $i=1, \ldots, N$ and $S \subset\{1, \ldots, N\}$ with $i \notin S$ let

$$
u_{S}=\min _{s \in S}\left\{u_{s}, v\right\} \quad \text { and } \quad \varphi_{i, S}=\left(u_{S}-u_{i}\right)_{+} .
$$

Then $0 \leq \varphi_{i, S} \leq \varphi$ and hence $\varphi_{i, S} \in W_{0}^{1, p}(\Omega)$. (Note that $\varphi_{i, S}=0$ if $i \in S$.) Testing (1.1) for each $u_{i}$ with $\varphi_{i, S}$ provides us with $N 2^{N-1}$ inequalities of the form

$$
\int_{u_{i}<u_{S}}\left|\nabla u_{i}\right|^{p} d x \leq Q_{i} \int_{u_{i}<u_{S}}\left|\nabla u_{S}\right|^{p} d x .
$$

This leads to a linear programming problem, which is solvable in polynomial time with respect to the number of the conditions.

Remark 4.1. When formulating the linear programming problem one can without loss of generality assume that the sets $\left\{x \in \Omega: u_{i}(x)=u_{j}(x)\right\}, 1 \leq i<j \leq N$, all have measure zero; this follows from the fact that we can approximate each $u_{i}$ from below using $u_{i}-q_{i}$, with rational $q_{i} \geq 0$, and the corresponding minima increase to $u$, while preserving the quasisuperminimizing constant, by Theorem 6.1 in Kinnunen-Martio [21].

For example, when $N=3$, we obtain 12 conditions. We used Mathematica to solve this linear programming problem and obtained the following result. Below we provide a direct proof without relying on Mathematica. However, the Mathematica calculation shows that the constant obtained here is the best possible using only the information above.

Theorem 4.2. Let $u_{i}$ be a $Q_{i}$-quasisuperminimizer for $i=1,2,3$. Let

$$
P=2 Q_{1} Q_{2} Q_{3}-Q_{1} Q_{2}-Q_{2} Q_{3}-Q_{3} Q_{1}+1
$$

and, with $\{j, k\}=\{1,2,3\} \backslash\{i\}$,

$$
R_{i}= \begin{cases}0, & \text { if } Q_{j}=Q_{k}=1 \\ \frac{\left(Q_{j}-1\right)\left(Q_{k}-1\right)\left(Q_{j}-1+Q_{k}-1\right)}{Q_{j} Q_{k}-1}, & \text { otherwise. }\end{cases}
$$

Then $\min \left\{u_{1}, u_{2}, u_{3}\right\}$ is a $\bar{Q}$-quasisuperminimizer with

$$
\bar{Q}=\frac{Q_{1} Q_{2} Q_{3}}{P}\left(R_{1}+R_{2}+R_{3}\right)
$$

unless at least two of the $Q_{i}$ equal 1 , say $Q_{2}=Q_{3}=1$, in which case $\bar{Q}=Q_{1}$.

It is easily verified that the choice $Q_{3}=1$ gives the expression in Theorem 1.2. When $Q_{1}=Q_{2}=Q_{3}$, it is also easy to verify that the constant gets the following simpler form. 
Corollary 4.3. Let $u_{1}, u_{2}, u_{3}$ be $Q$-quasisuperminimizers. Then $\min \left\{u_{1}, u_{2}, u_{3}\right\}$ is a $6 Q^{3} /(Q+1)(2 Q+1)$-quasisuperminimizer.

This estimate is slightly better than what we would have obtained by iterating Theorem 1.2: First, the minimum of $u_{1}$ and $u_{2}$ is a $2 Q^{2} /(Q+1)$-quasisuperminimizer, and then the minimum of $\min \left\{u_{1}, u_{2}\right\}$ and $u_{3}$, i.e. the minimum of a $2 Q^{2} /(Q+1)$ and a $Q$-quasisuperminimizer, is a $2 Q^{3}(3 Q+2) /(Q+1)\left(2 Q^{2}+2 Q+1\right)$-quasisuperminimizer, by Theorem 1.2. However both of these estimates give values close to $3 Q$ for large values of $Q$.

We now explain how Theorem 4.2 can be proved without the use of Mathematica.

Proof of Theorem 4.2. If $\min \left\{Q_{1}, Q_{2}, Q_{3}\right\}=1$, we have already noticed that the result follows from Theorem 1.2 , so we assume that $\min \left\{Q_{1}, Q_{2}, Q_{3}\right\}>1$.

The proof is similar to the proof of Theorem 1.2, it just requires more book keeping. There are 12 inequalities of the form (4.1) at our disposal. More precisely, for $S=\varnothing$, there are three inequalities

$$
\int_{u_{i}<v}\left|\nabla u_{i}\right|^{p} d x \leq Q_{i} \int_{u_{i}<v}|\nabla v|^{p} d x
$$

$i=1,2,3$. For singleton $S=\{j\}, j \neq i$, we obtain six possible inequalities, namely

$$
\int_{u_{i}<\min \left\{u_{j}, v\right\}}\left|\nabla u_{i}\right|^{p} d x \leq Q_{i} \int_{u_{i}<\min \left\{u_{j}, v\right\}}\left|\nabla \min \left\{u_{j}, v\right\}\right|^{p} d x
$$

$i, j=1,2,3, i \neq j$. Finally, for $S=\{j, k\}, i \notin S$, we have three inequalities

$$
\int_{u_{i}<\min \left\{u_{j}, u_{k}, v\right\}}\left|\nabla u_{i}\right|^{p} d x \leq Q_{i} \int_{u_{i}<\min \left\{u_{j}, u_{k}, v\right\}}\left|\nabla \min \left\{u_{j}, u_{k}, v\right\}\right|^{p} d x,
$$

$i=1,2,3$.

Depending on the choice of the set $S$ and on the sizes of the functions $u_{1}, u_{2}$, $u_{3}$ and $v$, the sets of integration in these equations split into three different sets, where also $u_{S}=\min _{s \in S}\left\{u_{s}, v\right\}$ equals different $u_{i}$ or $v$.

Let $\pi=(i j k)$ be a fixed but arbitrary permutation of the set $\{1,2,3\}$. Then the following subsets of the set $A=\left\{x \in \Omega: \varphi(x)>0\right.$ and $\left.u_{i}(x)<u_{j}(x)<u_{k}(x)\right\}$ are of interest:

$$
\begin{aligned}
& A_{0}=\left\{x \in A: u_{i}(x)<u_{j}(x)<u_{k}(x)<v(x)\right\}, \\
& A_{1}=\left\{x \in A: u_{i}(x)<u_{j}(x)<v(x)<u_{k}(x)\right\}, \\
& A_{2}=\left\{x \in A: u_{i}(x)<v(x)<u_{j}(x)<u_{k}(x)\right\} .
\end{aligned}
$$

(Note that by Remark 4.1, we can assume that all the sets $\left\{x \in \Omega: u_{i}(x)=u_{j}(x)\right\}$ have measure zero.) We shall now check in which of the above inequalities these sets appear as parts of the sets of integration. We shall also keep track of which function then appears in the left-hand side (LHS) and in the right-hand side (RHS). It is immediate that none of $A_{0}, A_{1}$ and $A_{2}$ is present in the equations $\left(E_{j i}\right),\left(E_{k i}\right)$, $\left(E_{k j}\right),\left(\widehat{E}_{j}\right)$ or $\left(\widehat{E}_{k}\right)$. The set $A_{2}$ appears only in $\left(E_{i}\right),\left(E_{i j}\right),\left(E_{i k}\right)$ and $\left(\widehat{E}_{i}\right)$, and the function in the LHS is then always $u_{i}$, while the one in the RHS is always $v$. For the sets $A_{0}$ and $A_{1}$, the choices of funtions are more complicated and are summarized in Table 3 .

We multiply the inequalities $\left(E_{i}\right),\left(E_{i j}\right)$ and $\left(\widehat{E}_{i}\right)$ by $x_{i}, x_{i j}$ and $\hat{x}_{i}$, respectively, and sum up. We have $u=u_{i}$ everywhere in the set $A=A_{0} \cup A_{1} \cup A_{2}$, and hence to show that $u$ is a quasisuperminimizer, we need to keep track of $\int_{A}\left|\nabla u_{i}\right|^{p} d x$ in the LHSs and of $\int_{A}|\nabla v|^{p} d x$ in the RHSs. We also want to choose $x_{i}, x_{i j}$ and $\hat{x}_{i}$ so 


\begin{tabular}{|c|c||c|c||c|}
\hline $\begin{array}{c}\text { Integral } \\
\text { over the set }\end{array}$ & $\begin{array}{c}\text { Appears in } \\
\text { the inequality }\end{array}$ & $\begin{array}{c}\text { Gradient } \\
\text { in the LHS }\end{array}$ & $\begin{array}{c}\text { Gradient } \\
\text { in the RHS }\end{array}$ & $\begin{array}{c}\text { Constant } \\
\text { in the RHS }\end{array}$ \\
\hline \hline$A_{0}$ & $\left(E_{i}\right)$ & $\nabla u_{i}$ & $\nabla v$ & $Q_{i}$ \\
\hline$A_{0}$ & $\left(E_{j}\right)$ & $\nabla u_{j}$ & $\nabla v$ & $Q_{j}$ \\
\hline$A_{0}$ & $\left(E_{k}\right)$ & $\nabla u_{k}$ & $\nabla v$ & $Q_{k}$ \\
\hline$A_{0}$ & $\left(E_{i j}\right)$ & $\nabla u_{i}$ & $\nabla u_{j}$ & $Q_{i}$ \\
\hline$A_{0}$ & $\left(E_{i k}\right)$ & $\nabla u_{i}$ & $\nabla u_{k}$ & $Q_{i}$ \\
\hline$A_{0}$ & $\left(E_{j k}\right)$ & $\nabla u_{j}$ & $\nabla u_{k}$ & $Q_{j}$ \\
\hline$A_{0}$ & $\left(\widehat{E}_{i}\right)$ & $\nabla u_{i}$ & $\nabla u_{j}$ & $Q_{i}$ \\
\hline \hline$A_{1}$ & $\left(E_{i}\right)$ & $\nabla u_{i}$ & $\nabla v$ & $Q_{i}$ \\
\hline$A_{1}$ & $\left(E_{j}\right)$ & $\nabla u_{j}$ & $\nabla v$ & $Q_{j}$ \\
\hline$A_{1}$ & $\left(E_{i j}\right)$ & $\nabla u_{i}$ & $\nabla u_{j}$ & $Q_{i}$ \\
\hline$A_{1}$ & $\left(E_{i k}\right)$ & $\nabla u_{i}$ & $\nabla v$ & $Q_{i}$ \\
\hline$A_{1}$ & $\left(E_{j k}\right)$ & $\nabla u_{j}$ & $\nabla v$ & $Q_{j}$ \\
\hline$A_{1}$ & $\left(\widehat{E}_{i}\right)$ & $\nabla u_{i}$ & $\nabla u_{j}$ & $Q_{i}$ \\
\hline \hline$A_{2}$ & $\left(E_{i}\right)$ & $\nabla u_{i}$ & $\nabla v$ & $Q_{i}$ \\
\hline$A_{2}$ & $\left(E_{i j}\right)$ & $\nabla u_{i}$ & $\nabla v$ & $Q_{i}$ \\
\hline$A_{2}$ & $\left(E_{i k}\right)$ & $\nabla u_{i}$ & $\nabla v$ & $Q_{i}$ \\
\hline$A_{2}$ & $\left(\widehat{E}_{i}\right)$ & $\nabla u_{i}$ & $\nabla v$ & $Q_{i}$ \\
\hline
\end{tabular}

Table 3.

that the integrals of $\left|\nabla u_{j}\right|^{p}$ and $\left|\nabla u_{k}\right|^{p}$ in the RHSs are compensated by the same integrals in the LHSs.

From Table 3 , we see that $\nabla u_{j}$ cancels out in $A_{0}$ and $A_{1}$ if we have

$$
x_{j}+x_{j k}-Q_{i} x_{i j}-Q_{i} \hat{x}_{i}=0,
$$

and that $\nabla u_{k}$ cancels out in $A_{0}$, if

$$
x_{k}-Q_{i} x_{i k}-Q_{j} x_{j k}=0
$$

In addition, we want the coefficients in front of the terms containing $\nabla u_{i}=\nabla u$ in each of the sets $A_{0}, A_{1}$ and $A_{2}$ to sum up to 1 , i.e.

$$
x_{i}+x_{i j}+x_{i k}+\hat{x}_{i}=1 \text {. }
$$

Considering all permutations of $\{1,2,3\}$ we obtain a linear system of 12 equations with 12 unknowns. However, the system can be simplified, which we do now. From (4.4) we obtain $\hat{x}_{i}=1-\left(x_{i}+x_{i j}+x_{i k}\right)$ and inserting this into (4.2) gives

$$
x_{j}+x_{j k}+Q_{i} x_{i}+Q_{i} x_{i k}=Q_{i}
$$

From (4.3) we have $Q_{i} x_{i k}=x_{k}-Q_{j} x_{j k}$, which together with (4.5) leads to

$$
Q_{i} x_{i}+x_{j}+x_{k}+\left(1-Q_{j}\right) x_{j k}=Q_{i} .
$$


Now, note that this equation is for fixed $i$ symmetric in $j$ and $k$, except for the last term in the left-hand side, which thus must be symmetric in $j$ and $k$ as well. Hence, we see that

$$
\left(1-Q_{j}\right) x_{j k}=\left(1-Q_{k}\right) x_{k j}=: y_{i}
$$

Thus the above system transforms into the six equations

$$
\begin{aligned}
x_{k}+S_{i} y_{j}+S_{j} y_{i} & =0, \\
Q_{i} x_{i}+x_{j}+x_{k}+y_{i} & =Q_{i},
\end{aligned}
$$

where $S_{i}=Q_{i} /\left(Q_{i}-1\right)$. It can be written as

$$
\left\{\begin{array}{l}
x+S y=0, \\
R x+y=c,
\end{array} \quad \text { with } \quad x=\left(\begin{array}{l}
x_{1} \\
x_{2} \\
x_{3}
\end{array}\right) \quad \text { and } \quad y=\left(\begin{array}{l}
y_{1} \\
y_{2} \\
y_{3}
\end{array}\right)\right.
$$

where

$$
S=\left(\begin{array}{ccc}
0 & S_{3} & S_{2} \\
S_{3} & 0 & S_{1} \\
S_{2} & S_{1} & 0
\end{array}\right), \quad R=\left(\begin{array}{ccc}
Q_{1} & 1 & 1 \\
1 & Q_{2} & 1 \\
1 & 1 & Q_{3}
\end{array}\right) \quad \text { and } \quad c=\left(\begin{array}{l}
Q_{1} \\
Q_{2} \\
Q_{3}
\end{array}\right)
$$

From the second equation we have $y=c-R x$, which transforms the first equation into $(S R-I) x=S c$, whose solution is

$$
x=(S R-I)^{-1} S c,
$$

where $I$ stands for the identity matrix.

Now, as we have chosen $x$ (and thus $y$ ), so that all extra terms in the equations $\left(E_{i}\right),\left(E_{i j}\right),\left(\widehat{E}_{i}\right), i, j=1,2,3$, cancel out and the remaining ones with $\nabla u$ always appear with coefficient 1 in the LHS, we need to check how large constants appear with $|\nabla v|^{p}$ in the RHS to determine $\bar{Q}$. From Table 3, we see that $\int_{A_{0}}|\nabla v|^{p} d x$ appears in the right-hand side with a factor

$$
Q_{A_{0}}=Q_{i} x_{i}+Q_{j} x_{j}+Q_{k} x_{k}
$$

Similarly, the factors are

$$
\begin{array}{ll}
Q_{A_{1}}=Q_{i} x_{i}+Q_{j} x_{j}+Q_{i} x_{i k}+Q_{j} x_{j k}=Q_{i} x_{i}+Q_{j} x_{j}+x_{k} \leq Q_{A_{0}} & \text { (by (4.3)), } \\
Q_{A_{2}}=Q_{i}\left(x_{i}+x_{i j}+x_{i k}+\hat{x}_{i}\right)=Q_{i} & \text { (by (4.4)), }
\end{array}
$$

for $\int_{A_{1}}|\nabla v|^{p} d x$ and $\int_{A_{2}}|\nabla v|^{p} d x$, respectively. Since the quasiminimizing constant $\bar{Q}$ of $u$ must be at least $\max \left\{Q_{1}, Q_{2}, Q_{3}\right\}$, we conclude that $Q_{A_{0}}$ is the largest of the three and

$$
\bar{Q}=Q_{A_{0}}=c^{T} x=c^{T}(S R-I)^{-1} S c=\left(\left((S R-I)^{T}\right)^{-1} c\right)^{T} S c
$$

where ${ }^{T}$ denotes the matrix transpose. Observe that the value of $Q_{A_{0}}$ is symmetric in $i, j$ and $k$. An elementary calculation shows that

$S R-I=\left(\begin{array}{ccc}L_{1} & Q_{2} L_{1} & Q_{3} L_{1} \\ Q_{1} L_{2} & L_{2} & Q_{3} L_{2} \\ Q_{1} L_{3} & Q_{2} L_{3} & L_{3}\end{array}\right)$, where $L_{i}=S_{j}+S_{k}-1=\frac{Q_{j} Q_{k}-1}{\left(Q_{j}-1\right)\left(Q_{k}-1\right)}$,

for $i \neq j \neq k \neq i$. Thus, $z:=\left((S R-I)^{T}\right)^{-1} c$ is the unique solution of the system $(S R-I)^{T} z=c$, which can be equivalently written as

$$
\left(\begin{array}{ccc}
\frac{L_{1}}{Q_{1}} & L_{2} & L_{3} \\
L_{1} & \frac{L_{2}}{Q_{2}} & L_{3} \\
L_{1} & L_{2} & \frac{L_{3}}{Q_{3}}
\end{array}\right)\left(\begin{array}{l}
z_{1} \\
z_{2} \\
z_{3}
\end{array}\right)=\left(\begin{array}{l}
1 \\
1 \\
1
\end{array}\right)
$$


Denoting the matrix in the left-hand side by $L$, Cramer's rule gives

$$
z_{i}=\frac{L_{j} L_{k}}{Q_{j} Q_{k} \operatorname{det} L}\left(Q_{j}-1\right)\left(Q_{k}-1\right), \quad i \neq j \neq k \neq i
$$

where

$$
\operatorname{det} L=\frac{L_{1} L_{2} L_{3}}{Q_{1} Q_{2} Q_{3}}\left(2 Q_{1} Q_{2} Q_{3}-Q_{1} Q_{2}-Q_{2} Q_{3}-Q_{3} Q_{1}+1\right)=: \frac{L_{1} L_{2} L_{3}}{Q_{1} Q_{2} Q_{3}} P .
$$

It follows that $z_{i}=Q_{i}\left(Q_{j}-1\right)\left(Q_{k}-1\right) / L_{i} P$. We also have $S c=w$, where

$$
w_{i}=Q_{j} S_{k}+Q_{k} S_{j}=Q_{j} Q_{k}\left(\frac{1}{Q_{j}-1}+\frac{1}{Q_{k}-1}\right),
$$

and hence,

$$
\begin{aligned}
w_{i} z_{i} & =Q_{j} Q_{k}\left(\frac{1}{Q_{j}-1}+\frac{1}{Q_{k}-1}\right) \frac{Q_{i}}{L_{i} P}\left(Q_{j}-1\right)\left(Q_{k}-1\right) \\
& =\frac{Q_{1} Q_{2} Q_{3}}{P} \frac{\left(Q_{j}-1\right)\left(Q_{k}-1\right)\left(Q_{j}-1+Q_{k}-1\right)}{Q_{j} Q_{k}-1}=: \frac{Q_{1} Q_{2} Q_{3}}{P} R_{i} .
\end{aligned}
$$

Consequently, going back to (4.6) we obtain

$$
\bar{Q}=Q_{A_{0}}=\sum_{i=1}^{3} w_{i} z_{i}=\frac{Q_{1} Q_{2} Q_{3}}{P}\left(R_{1}+R_{2}+R_{3}\right) .
$$

\section{Blowup in pasting lemmas}

In this section we shall show that the quasisuperminimizing constant $Q_{1} Q_{2}$ in the pasting Theorem 1.4 is optimal. More precisely, we prove the following result.

Theorem 5.1. Let $p, Q_{1}$ and $Q_{2}$ be given. Then there are $u_{1}, u_{2}$ and open sets $\Omega_{1} \subset \Omega_{2}$, such that $u_{j}$ is a $Q_{j}$-quasiminimizer in $\Omega_{j}, j=1,2$, and

$$
u= \begin{cases}u_{2}, & \text { in } \Omega_{2} \backslash \Omega_{1}, \\ \min \left\{u_{1}, u_{2}\right\}, & \text { in } \Omega_{1},\end{cases}
$$

is a quasisuperminimizer in $\Omega_{2}$ with the optimal quasisuperminimizer constant $Q_{1} Q_{2}$.

This is in sharp contrast to Theorem 1.2 , where $\min \left\{u_{1}, u_{2}\right\}$ is guaranteed to have a quasisuperminimizing constant $\widetilde{Q}<Q_{1} Q_{2}$, and moreover,

$$
Q_{1} Q_{2}-\widetilde{Q}=Q_{1} Q_{2} \frac{\left(Q_{1}-1\right)\left(Q_{2}-1\right)}{Q_{1} Q_{2}-1}>0 \quad \text { whenever } Q_{1}, Q_{2}>1
$$

A drawback of our proof of Theorem 5.1 is that $\Omega_{1}$ is not connected. However even when $\Omega_{1}$ is required to be connected we can show, by varying $p$, the optimality of the blowup constant in Theorem 1.4 using the following result.

Theorem 5.2. Let $Q_{1}, Q_{2}$ and $\varepsilon>0$ be given. Then there are $p, u_{1}, u_{2}$ and an interval $I=\left(x_{0}, 1\right), 0 \leq x_{0}<1$, such that $u_{1}$ is a $Q_{1}$-quasiminimizer in $I, u_{2}$ is a $Q_{2}$-quasiminimizer in $\Omega=(0,1)$, and

$$
u= \begin{cases}u_{2}, & \text { in } \Omega \backslash I, \\ \min \left\{u_{1}, u_{2}\right\}, & \text { in } I,\end{cases}
$$

is a $Q$-quasisuperminimizer in $\Omega$ with optimal quasisuperminimizer constant

$$
Q \geq Q_{1} Q_{2}-\varepsilon
$$


Remark 5.3. The functions $u_{1}, u_{2}$ and $u$ in the proofs below of Theorems 5.1 and 5.2 are continuous, and hence this also demonstrates the sharpness of the blowup in the pasting lemma for quasisuperharmonic functions (Theorem 5.1 in A. BjörnMartio [9]).

To prove these theorems we need to use some results on one-corner functions. In particular, we will use the following result which was obtained by Uppman [34, Section 2.2.3]. For $p=2$ it is due to Judin [18, Example 4.0.25].

Theorem 5.4. Let $0<\alpha<\beta<\infty$ and $\gamma=\beta / \alpha$. The optimal quasiminimizer constant for

$$
u(x)= \begin{cases}\alpha x, & x \leq 0 \\ \beta x, & x \geq 0\end{cases}
$$

$i s$

$$
Q=\frac{\left(\gamma^{p}+k\right)(1+k)^{p-1}}{(\gamma+k)^{p}}
$$

where

$$
k=\frac{p \gamma^{p}(\gamma-1)-\gamma\left(\gamma^{p}-1\right)}{\gamma^{p}-1-p(\gamma-1)},
$$

Moreover $u$ has the maximal p-energy allowed by $Q$ on an interval of the form $[-a, b], a, b>0$, if and only if $a / b=k$.

The last part is a consequence of the proof by Uppman (or Judin in the case when $p=2$ ). Recall from Definition 3.3 that a quasiminimizer is said to have the maximal $p$-energy allowed by $Q$ on an interval $I$ if its $p$-energy therein is $Q$-times the $p$-energy of the linear function with the same boundary values on $\partial I$. Note also that $k=\gamma$ if $p=2$.

We will say that $u$ as in (5.3) is a one-corner function with corner 0 and quotient $\gamma$. We will mainly be interested in convex one-corner functions as these are subminimizers and thus $Q$ above is also the optimal quasisuperminimizer constant.

Proposition 5.5. The function $Q(\gamma, p)$ is continuous, and moreover it is strictly increasing with respect to $\gamma$.

Proof. The continuity follows directly from the expressions in Theorem 5.4.

Let $\gamma^{\prime}>\gamma$ and let $I=[-a, 1]$ be an interval such that $u$ has the maximal $p$ energy allowed by $Q$ on $I$, where $u$ and $Q$ are given by Theorem 5.4 with $\gamma=\beta / \alpha$. Let $\beta^{\prime}=\gamma^{\prime} \alpha>\beta$. Choose $0<x_{0}<1$ so that $\alpha x_{0}+\beta^{\prime}\left(1-x_{0}\right)=\beta$ and let

$$
w(x)= \begin{cases}\alpha x, & x \leq x_{0}, \\ \beta^{\prime}\left(1-x_{0}\right)+\beta, & x \geq x_{0} .\end{cases}
$$

Then $w$ is a $Q^{\prime}=Q\left(\gamma^{\prime}, p\right)$-quasiminimizer in $I, w=u$ on $[-a, 0] \cup\{1\}$ and $w<u$ in $(0,1)$. Hence, if $v$ is the linear function in $I$ with boundary values $v=w$ on $\partial I$, then

$$
\int_{I}\left|w^{\prime}\right|^{p} d x>\int_{I}\left|u^{\prime}\right|^{p} d x=Q \int_{I}\left|v^{\prime}\right|^{p} d x
$$

This shows that $Q^{\prime}>Q$.

A direct consequence of Proposition 5.5 is that we can view $\gamma$ as a function of $Q$ and $p$, and this function is strictly increasing with respect to $Q$. We will also need the following estimate.

Proposition 5.6. It is always true that $Q \leq \gamma^{p-1}$. 
Proof. Let $c=k / \gamma$. Then

$$
Q=\frac{\left(\gamma^{p}+k\right)(1+k)^{p-1}}{(\gamma+k)^{p}} \leq \frac{\gamma^{p}+k}{\gamma+k}=\frac{\gamma^{p}+c \gamma}{(1+c) \gamma} \leq \frac{\gamma^{p}+c \gamma^{p}}{(1+c) \gamma}=\gamma^{p-1}
$$

Remark 5.7. A direct calculation of $\gamma^{p}+k, 1+k$ and $\gamma+k$ yields after simplifications that

$$
Q=\frac{(p-1)^{p-1}\left(\gamma^{p}-1\right)^{p}}{p^{p}\left(\gamma^{p}-\gamma\right)^{p-1}(\gamma-1)}=\frac{(p-1)^{p-1}}{p^{p}}\left(\frac{\gamma^{p}-1}{\gamma^{p}-\gamma}\right)^{p-1} \frac{\gamma^{p}-1}{\gamma-1}
$$

It is easily verified that $\gamma^{p}-1 \geq(\gamma-1) \gamma^{p-1}$, and inserting this into (5.4) gives, together with Proposition 5.6, the two-sided estimate

$$
Q \leq \gamma^{p-1} \leq \frac{p^{p} Q}{(p-1)^{p-1}}
$$

Lemma 5.8. Given $\gamma>1$, let $Q$ be as in Theorem 5.4. Then the function

$$
u(x)= \begin{cases}\alpha x, & 0<x \leq x_{0} \\ 1+\alpha \gamma(x-1), & x_{0} \leq x<1\end{cases}
$$

with

$$
x_{0}=\frac{p \gamma^{p}(\gamma-1)-\gamma\left(\gamma^{p}-1\right)}{(p-1)\left(\gamma^{p}-1\right)(\gamma-1)} \quad \text { and } \quad \alpha=\frac{p-1}{p} \frac{\gamma^{p}-1}{\gamma^{p}-\gamma},
$$

is the unique one-corner function with the boundary conditions $u(0)=0$ and $u(1)=$ 1 that is convex and has the maximal p-energy allowed by $Q$ on $(0,1)$.

Proof. For $u$ to be continuous, it is required that $\alpha x_{0}+\alpha \gamma\left(1-x_{0}\right)=1$, i.e. that

$$
\alpha=\frac{1}{\gamma+x_{0}(1-\gamma)}
$$

Theorem 5.4 with $a=x_{0}$ and $b=1-x_{0}$ gives $x_{0} /\left(1-x_{0}\right)=k$, i.e. $x_{0}=k /(k+1)$. The formula for $k$ from Theorem 5.4 then yields after some simplification the formula for $x_{0}$ in (5.6). Inserting that into (5.7) then concludes the proof of the lemma, since uniqueness follows by construction.

Remark 5.9. Lemma 5.8 can also be proved without an appeal to Theorem 5.4 by maximizing the $p$-energy

$$
E=\int_{0}^{1}\left|u_{2}^{\prime}\right|^{p} d x=\alpha^{p} x_{0}+\alpha^{p} \gamma^{p}\left(1-x_{0}\right)=\frac{x_{0}+\gamma^{p}\left(1-x_{0}\right)}{\left(\gamma+x_{0}(1-\gamma)\right)^{p}}
$$

with respect to $x_{0}$.

Remark 5.10. A straightforward calculation shows that for $Q$ and $\alpha$ from (5.4) and (5.7) it holds that $Q=Q_{\alpha}=Q_{\alpha \gamma}$, where $Q_{\alpha}$ and $Q_{\alpha \gamma}$ are related to $\alpha$ and $\alpha \gamma$ as in (3.1). Thus, the optimal one-corner function provided by Lemma 5.8 is tangent at the end points 1 and 0 to the power-like functions $u_{Q}$ and $\bar{u}_{Q}$ from (3.2), respectively.

The proof below of Theorem 5.2 is based on varying $p$ and the fact that the constant in Theorem 1.4 is independent of $p$. For fixed $p$ we obtain the following somewhat weaker result. 
Proposition 5.11. Let $p, Q_{1}$ and $Q_{2}$ be given. Then there are $u_{1}, u_{2}$ and an interval $I=\left(x_{0}, 1\right), x_{0} \geq 0$, such that $u_{1}$ is a $Q_{1}$-quasiminimizer in $I, u_{2}$ is a $Q_{2}$-quasiminimizer in $\Omega=(0,1)$, and

$$
u= \begin{cases}u_{2}, & \text { in } \Omega \backslash I, \\ \min \left\{u_{1}, u_{2}\right\}, & \text { in } I .\end{cases}
$$

is a $Q$-quasisuperminimizer in $\Omega$ with optimal quasisuperminimizer constant

$$
Q \geq Q_{1}\left(Q_{2}-1\right)+1=Q_{1} Q_{2}-Q_{1}+1
$$

If moreover $Q_{1}>1$, then the inequality in (5.8) is strict, i.e. $Q>Q_{1}\left(Q_{2}-1\right)+1$.

Proof. Using Proposition 5.5 and Lemma 5.8 we can find $0 \leq x_{0}<1, \gamma \geq 1$ and $0<\alpha \leq 1$ such that the function $u_{2}$ given by $(5.5)$ is a $Q_{2}$-quasiminimizer in $\Omega$ with the maximal $p$-energy allowed by $Q_{2}$ on $\Omega$. For $Q_{2}=1$ let $u_{2}(x)=x$ and $x_{0}=0$.

Another use of Lemma 5.8 provides us with a convex one-corner function $u_{1}$ which is a $Q_{1}$-quasiminimizer in $I=\left(x_{0}, 1\right)$ with boundary values $u_{1}=u_{2}$ on $\partial I$ and maximal $p$-energy allowed by $Q_{1}$ on $I$. (If $Q_{1}=1$, we let $u_{1} \equiv u_{2}$ on $I$, which is not a one-corner function.)

Since $\alpha \leq 1$ and $x_{0}<1$, we have

$$
A:=\int_{0}^{x_{0}}\left|u_{2}^{\prime}\right|^{p} d x=\alpha^{p} x_{0}<1
$$

It then follows that

$$
\begin{aligned}
\int_{0}^{1}\left|u^{\prime}\right|^{p} d x & =\int_{0}^{x_{0}}\left|u_{2}^{\prime}\right|^{p} d x+\int_{x_{0}}^{1}\left|u_{1}^{\prime}\right|^{p} d x=A+Q_{1} \int_{x_{0}}^{1}\left|u_{2}^{\prime}\right|^{p} d x \\
& =A+Q_{1}\left(Q_{2}-A\right)=Q_{1} Q_{2}-A\left(Q_{1}-1\right) \geq Q_{1} Q_{2}-\left(Q_{1}-1\right),
\end{aligned}
$$

where the inequality is strict if $Q_{1}>1$. As $v(x)=x$ is the minimizer with boundary values $v=u$ on $\partial \Omega$, and its $p$-energy on $\Omega$ is 1 , this concludes the proof.

We are now ready to prove Theorems 5.1 and 5.2.

Proof of Theorem 5.1. The argument is a modification of the proof of Proposition 5.11. Let $\Omega_{2}=(0,1) \subset \mathbf{R}$ and $u_{2}$ and $x_{0}$ be as in the proof of Proposition 5.11. Now let $\Omega_{1}=\left(0, x_{0}\right) \cup\left(x_{0}, 1\right)$ and choose $u_{1}$ so that $u_{1}(x)=u_{2}(x)$ for $x=0, x_{0}, 1$, and its restrictions to $\left(0, x_{0}\right)$ and to $\left(x_{0}, 1\right)$ are convex one-corner functions provided by Lemma 5.8 , which are $Q_{1}$-quasiminimizers in the respective intervals and have the maximal energy therein allowed by $Q_{1}$. But then $u=u_{1}$ and

$$
\begin{aligned}
\int_{0}^{1}\left|u^{\prime}\right|^{p} d x & =\int_{0}^{x_{0}}\left|u_{1}^{\prime}\right|^{p} d x+\int_{x_{0}}^{1}\left|u_{1}^{\prime}\right|^{p} d x \\
& =Q_{1} \int_{0}^{x_{0}}\left|u_{2}^{\prime}\right|^{p} d x+Q_{1} \int_{x_{0}}^{1}\left|u_{2}^{\prime}\right|^{p} d x=Q_{1} \int_{0}^{1}\left|u_{2}^{\prime}\right|^{p} d x=Q_{1} Q_{2} .
\end{aligned}
$$

Proof of Theorem 5.2. We proceed as in the proof of Proposition 5.11. By Proposition 5.6, $1-\gamma^{1-p} \geq 1-1 / Q$. It thus follows from Lemma 5.8 that

$$
\alpha=\frac{p-1}{p} \frac{1-\gamma^{-p}}{1-\gamma^{1-p}}<\frac{p-1}{p} \frac{1}{1-1 / Q} \rightarrow 0, \quad \text { as } p \rightarrow 1+.
$$

Hence $A=\alpha^{p} x_{0}<\alpha^{p} \rightarrow 0$, as $p \rightarrow 1+$, so as in (5.9),

$$
\int_{0}^{1}\left|u^{\prime}\right|^{p} d x=Q_{1} Q_{2}-A\left(Q_{1}-1\right) \rightarrow Q_{1} Q_{2}, \quad \text { as } p \rightarrow 1+\text {. }
$$

(Note that $u_{1}, u_{2}$ and $u$ depend on $p$.) 
Remark 5.12. The estimate (5.8) in Proposition 5.11 can be replaced by $Q \geq$ $Q_{2}\left(Q_{1}+1\right) / 2$, which gives a better lower bound when $Q_{2}<2$. Indeed, we always have

$$
\int_{0}^{x_{0}}\left|u_{2}^{\prime}\right|^{p} d x \leq \frac{Q_{2}}{2} \quad \text { or } \quad \int_{x_{0}}^{1}\left|u_{2}^{\prime}\right|^{p} d x \leq \frac{Q_{2}}{2} .
$$

In the former case, the proof goes through as before, in the latter case, replace $u_{2}$ and $u_{1}$ by decreasing convex one-corner functions in $\Omega$ and $I$, respectively, with the maximal $p$-energies allowed by $Q_{2}$ and $Q_{1}$ therein, so that $u_{2}(0)=1, u_{1}\left(x_{0}\right)=$ $u_{2}\left(x_{0}\right)$ and $u_{1}(1)=u_{2}(1)=0$. In both cases, a direct calculation gives

$$
Q \geq Q_{1} Q_{2}-\frac{Q_{2}}{2}\left(Q_{1}-1\right)=\frac{Q_{2}\left(Q_{1}+1\right)}{2} .
$$

Corollary 5.13. Let p, $Q_{1}, Q_{2}$ and open sets $\Omega_{1} \varsubsetneqq \Omega_{2}=(0,1)$ be given. Then there are $u_{1}$ and $u_{2}$, which are $Q_{1}$ - and $Q_{2}$-quasiminimizers in $\Omega_{1}$ and $\Omega_{2}$, respectively, such that

$$
u= \begin{cases}u_{2}, & \text { in } \Omega_{2} \backslash \Omega_{1}, \\ \min \left\{u_{1}, u_{2}\right\}, & \text { in } \Omega_{1},\end{cases}
$$

is a $Q$-quasisuperminimizer in $\Omega$ with optimal quasisuperminimizer constant satisfying (5.8) and (5.10).

Proof. Since $\Omega_{1}$ is open, it can be written as a pairwise disjoint union of open intervals. Let $\left(x_{1}, x_{2}\right)$ be one of them and assume to begin with that $x_{1}>0$. We can then find $\delta>0$ so that

$$
\Omega^{\prime}:=\left(x_{1}-k \delta, x_{1}+\delta\right) \subset\left(0, x_{2}\right),
$$

where $k$ is the constant associated with $Q_{2}$ as in Theorem 5.4.

Rescale the functions in Proposition 5.11 or Remark 5.12 (depending on which gives a better estimate) so that they apply to the sets $\Omega^{\prime}$ and $I^{\prime}:=\left(x_{1}, x_{1}+\delta\right)$ in place of $\Omega$ and $I$. This provides us with one-corner functions $v_{1}$ and $v_{2}$, which are $Q_{1}$ - and $Q_{2}$-quasiminimizers in $I^{\prime}$ and $\Omega^{\prime}$, respectively, and their pasted function is a $Q$-quasisuperminimizer in $\Omega^{\prime}$ with optimal quasisuperminimizer constant $Q$ satisfying (5.8) and (5.10).

Now, let $u_{2}$ be the linear extension of $v_{2}$ which is a one-corner function on the whole of $(0,1)$. Also, let

$$
u_{1}= \begin{cases}u_{2}, & \text { in } \Omega_{1} \backslash\left(x_{1}, x_{2}\right), \\ v_{1}, & \text { in }\left(x_{1}, x_{2}\right),\end{cases}
$$

where $v_{1}$ is extended linearly as a one-corner functions on the whole of $\left(x_{1}, x_{2}\right)$. Then the best quasiminimizing constants of $u_{1}$ and $u_{2}$ in $\Omega_{1}$ and $\Omega_{2}$ are still $Q_{1}$ and $Q_{2}$, but their pasted function $u$ given by (5.11) will have its optimal quasisuperminimizing constant satisfying (5.8) and (5.10) in $\Omega^{\prime}$ and thus in $\Omega_{2}$.

If $x_{1}=0$ then necessarily $x_{2}<1$ and the above construction can be done for the interval $\left(1-x_{2}, 1\right)$ instead, replacing $u_{1}$ and $u_{2}$ by the decreasing convex one-corner functions $x \mapsto u_{1}(1-x)$ and $x \mapsto u_{2}(1-x)$.

We conclude the paper with further examples of quasiminimizers with explicit optimal quasiminimizing constants.

Proposition 5.14. Every strictly increasing continuous piecewise linear function $u$ in $(0,1)$ (having finitely many corners) with alternating slopes $\alpha$ and $\beta, \alpha<\beta$, is a quasiminimizer in $(0,1)$ with the best quasiminimizing constant $Q$ given by $(5.4)$ with $\gamma=\beta / \alpha$.

Moreover, if $u$ has at least one convex (concave) corner, then $Q$ is also the best quasisuperminimizing (quasisubminimizing) constant. 
Clearly, replacing $u$ with $x \mapsto u(1-x)$ gives a strictly decreasing quasiminimizer with the same best quasiminimizing constant as $u$. Note also that we do not require that the first segment defining $u$ has slope $\alpha$, nor that the last segment has slope $\beta$. However, we do not allow $u$ to be a linear function in the proposition, as then $\gamma=1$ and $Q$ cannot be defined using (5.4). Nevertheless, $Q=1$ is trivially the best quasiminimizing constant for $u$ in this case.

Proof. To show that $Q$ will do, let $0 \leq a<b \leq 1$ be arbitrary and consider the linear function $h$ with $h(a)=u(a)$ and $h(b)=u(b)$. By splitting $(a, b)$ into several subintervals, whose energies can be estimated separately, we may assume that either $h=u$ in $(a, b), h<u$ in $(a, b)$ or $h>u$ in $(a, b)$.

If $h>u$ in $(a, b)$, then moving from $a$ to $b$, we can successively eliminate the concave corners as follows: If

$$
u(x)=\max \left\{u\left(x^{\prime}\right)+\beta\left(x-x^{\prime}\right), u\left(x^{\prime \prime}\right)+\alpha\left(x-x^{\prime \prime}\right)\right\}
$$

in the interval $\left(x^{\prime}, x^{\prime \prime}\right)$, where $x^{\prime}$ and $x^{\prime \prime}$ are two convex corners, then replace $u$ in that interval by

$$
\min \left\{u\left(x^{\prime}\right)+\alpha\left(x-x^{\prime}\right), u\left(x^{\prime \prime}\right)+\beta\left(x-x^{\prime \prime}\right)\right\}
$$

This will decrease the number of corners in $(a, b)$ by 2 , while preserving the $p$-energy of $u$ therein. In the end, this procedure leaves us with a function which in $(a, b)$ coincides with a one-corner function $v$ with slopes $\alpha$ and $\beta$ and the same $p$-energy therein as $u$. Theorem 5.4 shows that $v$ is a $Q$-quasiminimizers in $(a, b)$ and hence

$$
\int_{a}^{b}\left|h^{\prime}\right|^{p} d x \leq Q \int_{a}^{b}\left|v^{\prime}\right|^{p} d x=Q \int_{a}^{b}\left|u^{\prime}\right|^{p} d x .
$$

The argument is similar when $h<u$ in $(a, b)$, while if $h=u$ in $(a, b)$ we trivially have $\int_{a}^{b}\left|h^{\prime}\right|^{p} d x=\int_{a}^{b}\left|u^{\prime}\right|^{p} d x<Q \int_{a}^{b}\left|u^{\prime}\right|^{p} d x$. As $a$ and $b$ were arbitrary, this shows that $u$ is a $Q$-quasiminimizer.

Finally, if $u$ has at least one convex (concave) corner, then considering intervals of type $\left(x_{0}-k \delta, x_{0}+\delta\right)$, where $x_{0}$ is one such corner, together with the last part of Theorem 5.4 shows that the quasisuperminimizing (quasisubminimizing) constant of $u$ cannot be better than $Q$. As every piecewise linear function with nonequal slopes has at least one corner, this concludes the proof.

\section{References}

1. BJörn, A., A weak Kellogg property for quasiminimizers, Comment. Math. Helv. 81 (2006), 809-825.

2. BJÖRN, A., Removable singularities for bounded $p$-harmonic and quasi(super)harmonic functions on metric spaces, Ann. Acad. Sci. Fenn. Math. 31 (2006), 71-95.

3. BJÖRn, A., A regularity classification of boundary points for $p$-harmonic functions and quasiminimizers, J. Math. Anal. Appl. 338 (2008), 39-47.

4. Buörn, A., Cluster sets for Sobolev functions and quasiminimizers, J. Anal. Math. 112 (2010), 49-77.

5. Björn, A. and Buörn, J., Power-type quasiminimizers, Ann. Acad. Sci. Fenn. Math. 36 (2011), 301-319.

6. BJörn, A. and BJörn, J., Nonlinear Potential Theory on Metric Spaces, EMS Tracts in Mathematics 17, European Math. Soc., Zürich, 2011. 
7. Buörn, A., Buörn, J. and MArola, N., BMO, local integrability, Harnack and Caccioppoli inequalities for quasisuperharmonic functions, Ann. Inst. H. Poincaré Anal. Non Linéaire 27 (2010), 1489-1505.

8. BJörn, A. and Marola, N., Moser iteration for (quasi)minimizers on metric spaces, Manuscripta Math. 121 (2006), 339-366.

9. Björn, A. and Martio, O., Pasting lemmas and characterizations of boundary regularity for quasiminimizers, Results Math. 55 (2009), 265-279.

10. BJörn, J., Boundary continuity for quasiminimizers on metric spaces, Illinois J. Math. 46 (2002), 383-403.

11. BJÖRN, J., Necessity of a Wiener type condition for boundary regularity of quasiminimizers and nonlinear elliptic equations, Calc. Var. Partial Differential Equations 35 (2009), 481-496.

12. BJÖRn, J., Sharp exponents and a Wiener type condition for boundary regularity of quasiminimizers, Preprint, 2015, arXiv:1504.08197.

13. DiBenedetto, E. and Gianazza, U., A Wiener-type condition for boundary continuity of quasi-minima of variational integrals, Preprint arXiv: 1504.01600 .

14. DiBenedetto, E. and Trudinger, N. S., Harnack inequalities for quasiminima of variational integrals, Ann. Inst. H. Poincaré Anal. Non Linéaire 1 (1984), 295-308.

15. Giaquinta, M. and Giusti, E., On the regularity of the minima of variational integrals, Acta Math. 148 (1982), 31-46.

16. Giaquinta, M. and Giusti, E., Quasi-minima, Ann. Inst. H. Poincaré Anal. Non Linéaire 1 (1984), 79-107.

17. Ivert, P.-A., Continuity of quasiminima under the presence of irregular obstacles, in Partial Differential Equations (Warsaw, 1984), pp. 155-167, Banach Center Publ., 19, PWN, Warsaw, 1987.

18. Judin, P. T., Onedimensional Quasiminimizers and Quasisuperminimizers [Yksiulotteiset Kvasiminimoijat ja Kvasisuperminimoijat], Licentiate thesis, Dept. of Math., Helsinki University, Helsinki, 2006 (Finnish).

19. Kinnunen, J., Kotilainen, M. and Latvala, V., Hardy-Littlewood type gradient estimates for quasiminimizers, Boll. Unione Mat. Ital. 3 (2010), $125-136$.

20. Kinnunen, J., Marola, N. and Martio, O., Harnack's principle for quasiminimizers, Ric. Mat. 56 (2007), 73-88.

21. Kinnunen, J. and Martio, O., Potential theory of quasiminimizers, Ann. Acad. Sci. Fenn. Math. 28 (2003), 459-490.

22. Kinnunen, J. and Shanmugalingam, N., Regularity of quasi-minimizers on metric spaces, Manuscripta Math. 105 (2001), 401-423.

23. Korte, R., Marola, N. and Shanmugalingam, N., Quasiconformality, homeomorphisms between metric measure spaces preserving quasiminimizers, and uniform density property, Ark. Mat. 50 (2012), 111-134.

24. Latvala, V., BMO-invariance of quasiminimizers., Ann. Acad. Sci. Fenn. Math. 29 (2004), 407-418.

25. Malý, J., Positive quasiminima, Comment. Math. Univ. Carolin. 24 (1983), 681-691.

26. Martio, O., Reflection principle for quasiminimizers, Funct. Approx. Comment. Math. 40 (2009), 165-173.

27. Martio, O., Quasiminimizers - definitions, constructions and capacity estimates, Lectures held at the conference Nonlinear problems for $\Delta_{p}$ and $\Delta$, Linköpings universitet, Linköping, 2009. http://www.mai.liu.se/TM/conf09/martio.pdf

28. Martio, O., Quasilinear Riccati type equations and quasiminimizers, Adv. Nonlinear Stud. 11 (2011), 473-482. 
29. Martio, O., Capacity and potential estimates for quasiminimizers, Complex Anal. Oper. Theory 5 (2011), 683-699.

30. Martio, O., Quasiminimizing properties of solutions to Riccati type equations, Ann. Sc. Norm. Super. Pisa Cl. Sci. 12 (2013), 823-832.

31. Martio, O. and Sbordone, C., Quasiminimizers in one dimension: integrability of the derivative, inverse function and obstacle problems, Ann. Mat. Pura Appl. 186 (2007), 579-590.

32. Moscariello, G., Weak minima and quasiminima of variational integrals, Boll. Un. Mat. Ital. B 11 (1997), 355-364.

33. Tolksdorf, P., Remarks on quasi(sub)minima, Nonlinear Anal. 10 (1986), $115-120$.

34. UpPman, H., The Reflection Principle for One-dimensional Quasiminimizers, Master's thesis, Linköpings universitet, Linköping, 2009.

http://urn.kb.se/resolve?urn=urn:nbn:se:liu:diva-19162

35. Ziemer, W. P., Boundary regularity for quasiminima, Arch. Ration. Mech. Anal. 92 (1986), 371-382. 